\title{
Experience on surgical treatment of gastrointestinal stromal tumor (GIST) of the stomach
}

\author{
E. ORSENIGO, P. GAZZETTA, S. DI PALO, A. TAMBURINI, C. STAUDACHER \\ Department of Surgery, University Vita-Salute San Raffaele, Milan, Italy
}

SUMMARY: Experience on surgical treatment of gastrointestinal stromal tumor (GIST) of the stomach.

\section{E. Orsenigo, P. Gazzetta, S. Di Palo, A. Tamburini, C. STAUDACHER}

We analyzed 75 cases of surgically treated gastric GIST. The surgical approach we employed was determined by tumor localization and size. 37,3\% of the patients underwent a laparoscopic procedure. No statistically significance in relapse and mortality rate was observed between laparoscopic and laparotomica group.

KEY WORDS: GIST, stomach, minimally invasive surgery.

\section{Aim}

We have retrospectively analyzed the hospital records of 75 patients who underwent a gastric surgical procedure for gastrointestinal stromal tumor between August 1994 and October 2008.

\section{Methods}

The patients were 37 males and 38 females with a mean age of 63 (SD 12). The class of risk (Miettinen/NIH), at the pathological evaluation of surgical specimen, was very-low in 12 patients $(16,4 \%)$, low in $23(31,5 \%)$, medium in $12(16,4 \%)$, and high in 26
$(35,6 \%)$. The mitotic count $(/ 50 \mathrm{HPF})$ was $<5$ in 51 pts (70,8\%), $6-10$ in 9 pts $(12,5 \%)$ and $>10$ in 12 pts $(16,7 \%)$, with a mean of $7,4 / 50 \mathrm{HPF}$ (sd 13,2). All the patients underwent gastric surgery. A conventional open approach was employed in 47 cases $(62,7 \%) ; 28$ patients $(37,3 \%)$ were treated with laparoscopic minimally invasive approach. The mean time of follow-up was 65 months (SD 50) (min 3- max 173 months).

\section{Results}

Relapse occurred in 10 patients (13,3\%) and metastatic spread in 10 pts $(13,3 \%)$. The relapse rate sorted by pathological risk was 6 in the medium/high risk group and 4 in the very low/low risk group. There was neither statistic difference in relapse rate sorted by classes of risk nor in relapse rate sorted by surgical approach (relapse rate: $9 \%$ in laparoscopy, $17,3 \%$ in laparotomy). 14 patients $(18,7 \%)$ have died ( 9 males, 5 females; 6 of them after GIST relapse).

\section{Conclusions}

In our experience, the laparoscopic approach to gastric GIST was feasible and safe.

Corrispondenza Autore:

Dott.ssa Elena Orsenigo

Dipartimento di Chirurgia e Specialistica

Università Vita-Salute San Raffaele

Via Olgettina, 60 - 20132 Milano

E-mail: orsenigo.elena @ hsr.it

(c) Copyright 2009, CIC Edizioni Internazionali, Roma 